\title{
Methylation and expression profiles in Apoe vicinity point to specific neighboring interaction of Apoe and TOMM40 genes: implication for the Alzheimer disease
}

\author{
Vladimir Babenko \\ Laboratory of Neuropathology \\ Modeling, Institute of Cytology and \\ Genetics, Siberian Branch of Russian \\ Academy of Sciences, Novosibirsk, \\ Russia \\ bob@bionet.nsc.ru
}

\author{
Roman Babenko \\ Institute of Cytology and Genetics, \\ Siberian Branch of Russian Academy \\ of Sciences, Novosibirsk, Russia \\ babe-roman@yandex.ru
}

\author{
Evgeny Rogaev \\ Institute of Basic Genetics, GSP-1 \\ Gubkina 3 Moscow, 11999, Russia \\ rogaev@vigg.ru
}

\begin{abstract}
We assessed the dynamics of 8 genes including TOMM40, Apoe and six adjacent ones for overall chromatin marks landscape, including methylation profiles across ENCODE brain cell lines, and histone and ctcf marks. We revealed the region manifests $\mathrm{Hi}-\mathrm{C}$ topology dynamics in a cellspecific manner. Additionally, based on methylation and histone marks profiles we underscore competitive manner of genes expression implying disrupted locus wide genes expression balance in Alzheimer patients due to Apoe extended locus methylation profile alteration.
\end{abstract}

Keywords - Alzheimer disease, methylation, aging, chromatin, GWAS

\section{Introduction}

As it is follows from its definition, Late Onset Alzheimeir Disease (LOAD) features late onset disease manifestation assuming some age - related processes are implicated. One of the factors could be well known age-related methylation profile alteration. Another specific to LOAD fact is the observation of extra high significance of SNPs, corresponding to Apoe Epsilon alleles, and LOAD linkage found in the successions of Genome Wide Association Studies (GWAS). That is why several recent studies $[1,2]$ explored association of epigenetic factors such DNA methylation and age and genotype in disease cohorts. Recent article [3] found significant differences in hippocampus and cerebellum methylation rate in TOMM40 promoter between LOAD patients' samples and controls. Also, evidence reported in [4] that besides Apoe gene, there is a range of SNPs within expanded Apoe related region significantly associated with LOAD risk, underlining PVRL2- CLPTM1 region is implicated in LOAD genetic risk rate.

\section{Research}

We scrutinized Apoe neighborhood and built the profiles of methylation and co-expression for 8 genes in the vicinity including Apoe. We observe specific pairwise correlation variation when comparing DNA methylation rate and particular gene expression. In particular, we elucidated complex 5' and 3' interplay across 8 adjacent genes region focused on Apoe, implying competitive expression of the neighbor genes depending on the methylation profile. As a conclusion, we report extensive cross-talk of methylation profile in the region, affecting the chromatin environment in the region, including ctcf and histone methylation marks.

\section{Conclusion}

We found that methylation profile alterations in Apoe region drastically change the balance of expression rates in genes of the region.

\section{Acknowledgment}

The work was supported by Russian Foundation for Basic Research grant 18-254-1764.

\section{References}

[1] Ma Y, Smith CE, Lai CQ, Irvin MR, Parnell LD, Lee YC, Pham L, Aslibekyan S, Claas SA, Tsai MY, Borecki IB, Kabagambe EK, Berciano S, Ordovás JM, Absher DM, Arnett DK. Genetic variants modify the effect of age on APOE methylation in the genetics of lipid lowering drugs and diet network study. Aging Cell. 2015;14(1):49-59. https://doi.org/10.1111/acel.12293.

[2] Babenko VN, Afonnikov DA, Ignatieva EV, Klimov AV, Gusev FE, Rogaev EI. Haplotype analysis of APOE intragenic SNPs. BMC Neurosci. 2018; 19(Suppl 1):16. doi: 10.1186/s12868-0180413-4

[3] Shao Y, Shaw M, Todd K, Khrestian M, D'Aleo G, Barnard PJ, Zahratka J, Pillai J, Yu CE, Keene CD, Leverenz JB, Bekris LM. DNA methylation of TOMM40-APOE-APOC2 in Alzheimer's disease. J Hum Genet. 2018 Apr;63(4):459-471. doi: 10.1038/s10038-017-0393-8. Epub 2018 Jan 25. PubMed PMID: 29371683; PubMed Central PMCID: PMC6466631.

[4] Zhou X, Chen Y, Mok KY, Kwok TCY, Mok VCT, Guo Q, Ip FC, Chen Y, Mullapudi N; Alzheimer's Disease Neuroimaging Initiative, Giusti-Rodríguez P, Sullivan PF, Hardy J, Fu AKY, Li Y, Ip NY. Non-coding variability at the APOE locus contributes to the Alzheimer's risk. Nat Commun. 2019 Jul 25;10(1):3310. doi 10.1038/s41467-019-10945-z. PubMed PMID: 31346172; PubMed Central PMCID PMC6658518. 